\title{
Polarization angle dependence of stark absorption spectra of spirilloxanthin bound to the reconstituted LH1 complexes using LH1-subunits isolated from the purple photosynthetic bacterium Rhodospirillum rubrum*
}

\author{
Tomoko Horibe1,2, Katsunori Nakagawa2,3, Toshiyuki Kusumoto1,2, Ritsuko Fujiï,3, Richard J. \\ Cogdell ${ }^{4}$, Mamoru Nango ${ }^{2,3}$ and Hideki Hashimoto ${ }^{1,2,3 凶}$
}

\begin{abstract}
'Department of Physics, Graduate School of Science, Osaka City University, Sugimoto, Sumiyoshi-ku, Osaka, Japan; ${ }^{2}$ CREST/JST, Honcho Kawaguchi, Saitama, Japan; ${ }^{T}$ The OCU Advanced Research Institute for Natural Science and Technology (OCARINA), Osaka City University, Sugimoto, Sumiyoshi-ku, Osaka, Japan; 4Department of Life and Materials Engineering, Graduate School of Engineering, Nagoya Institute of Technology, Gokiso-cho, Showa-ku, Nagoya, Japan; ${ }^{5}$ Glasgow Biomedical Research Centre, Faculty of Biomedical and Life Sciences, University of Glasgow, Glasgow, UK
\end{abstract}

Reconstituted LH1 complexes were prepared using the LH1 subunit-type complexes, isolated from the purple photosynthetic bacterium Rhodospirillum (Rs.) rubrum, and purified all-trans spirilloxanthin. Stark absorption spectra of spirilloxanthin bound to both the native and reconstituted LH1 complexes were compared in different polarization angles $(X)$ against the external electric field. From the polarization angle dependence of the Stark absorption spectra, two angles were determined in reference to the direction of transition dipole moment $(m)$ of spirilloxanthin: one is the change in polarizability upon photoexcitation $(\Delta a), \theta_{\Delta a}$ and the other is the change in static dipole moment upon photoexcitation $(\Delta \mu), \theta_{\Delta \mu}$. Despite the symmetric molecular structure of all-trans spirilloxanthin, its Stark absorption spectra show pronounced values of $\Delta \mu$. This large $\Delta \mu$ values essentially caused by the effect of induced dipole moment through $\Delta a$ both in the cases for native and reconstituted LH1 complexes. However, slightly different values of $\theta_{\Delta a}$ and $\theta_{\Delta \mu}$ observed for the native LH1 complex suggest that spirilloxanthin is asymmetrically distorted when bound to the native LH1 complex and gives rise to intrinsic $\Delta \mu$ value.

Key words: Stark absorption spectroscopy, polarization angle dependence, LH1 complex, reconstitution, Rhodospirillum rubrum, carotenoid, spirilloxanthin

Received: 14 October, 2011; accepted: 01 March, 2012; available on-line: 17 March, 2012

\section{INTRODUCTION}

The photosynthetic light-harvesting (LH) antenna absorbs light energy and transfers the captured excitation energy with high efficiency to the reaction centre (RC) $(\mathrm{Ke}, 2001)$. The LH system in photosynthetic purple bacteria generally consists of two types of antenna complexes, LH1 and LH2 (Cogdell et al., 2008). They are both oligomers of a simple monomeric unit consisting of a pair of low molecular weight, hydrophobic apo-proteins (called $\alpha$ and $\beta$ ). This pair of apo-proteins bind, non-covalently two or three bacteriochlorophyll $a$ $(\mathrm{BChl} a)$ and one carotenoid molecule. Optical properties of the pigments can be evaluated on the basis of the available structural data. From this perspective, LH2 whose structure has been determined in atomic resolu- tion served as a cornerstone for the development of a detailed understanding of structure-function relationships of the pigments in LH system (Cogdell et al., 2006). This is indeed a successful example to show the linkage of the structural biology and optical spectroscopy. However, when the detailed structural information has not yet been available, the optical spectroscopy still can afford the information about the structure of pigments bound to the LH complexes. This is exactly what we have done in this presented study for carotenoid bound to the LH1 complex from a purple photosynthetic bacterium. The only one successful X-ray crystal structure analysis for the LH1 complex has been reported for the LH1-RC complexes from a purple photosynthetic bacterium, Rhodopseudomonas (Rps.) palustris (Roszak et al., 2003). However, unfortunately the electron density that should be assignable for carotenoid has not yet been clarified with the reported $4.8 \AA$ resolution.

In order to draw out meaningful structural information using spectroscopy, we have applied Stark absorption spectroscopy to the carotenopid bound to the reconstituted LH1 complex from a purple photosynthetic bacterium, Rhodospirillum (Rsp.) rubrum. This particular spectroscopy is a well-established method with which to investigate the electrostatic properties of pigments embedded in photosynthetic pigment-protein complexes (Middendorf et al., 1993; Yanagi et al., 2004). Reconstitution of carotenoid into photosynthetic pigment-protein complexes has been proved to be powerful to study their functions (Akahane et al., 2004). This is partially because natural LH complexes always have contamination of the several different kinds of Car that are synthesized along the biosynthetic pathway. Hence, the reconstitution is the one possible way to prepare the $\mathrm{LH}$ complexes that have single carotenoid moiety. The LH1 complexes can be fully reconstituted in vitro from its constituent parts, i.e., the pigments (BChl $a$ and carotenoid) and, $\alpha$ - and $\beta$-apo-proteins (Iida et al., 2005; Cogdell et al., 2006). In the previous study (Nakagawa et al., 2008a), we have successfully reconstituted a series of carotenoids having dif-

e-mail address: hassy@sci.osaka-cu.ac.jp

* Presented at the 16th International Symposium on Carotenoids, 17-22 July, 2011, Kraków, Poland

Abbreviations: BChl, bacteriochlorophyll; LDAO, Lauryldimethylamine-oxide; LH, light-harvesting; PVA, Polyvinyl alcohol; RC, reaction centre; Rps., Rhodopseudomonas; Rsp., Rhodospirillum. 
ferent extent of conjugation into the LH1 complex of a purple photosynthetic bacterium Rsp. rubrum. The reconstituted LH1 complexes were investigated using absorption, fluorescence excitation, and Stark absorption spectroscopies. This study successfully correlates the nonlinear optical parameters determined by Stark absorption spectroscopy with those predicted by semi-empirical molecular orbital calculations and provides us with one of the promising strategies to discuss the conformation of the carotenoid molecules in the LH1 complexes. However, in this previous study, we have supposed that the spirilloxanthin bound to the reconstituted LH1 complex should have symmetric structure. This hypothesis has been tested in this present study.

In this study, we go one step further for the usage of the Stark absorption spectroscopy. The Stark absorption spectra were recorded in different polarization angles $(\chi)$ against the external electric field. From this set of measurements, we could determine the angles of both the change in polarizability upon photoexcitation $(\Delta \alpha)$ and the change in static dipole moment upon photoexcitation $(\Delta \mu)$ with respect to the transition dipole moment $(m)$ of carotenoid. Although the chemical structure of all-trans spirilloxanthin shown in Fig. 1 is symmetric, its symmetry breaking upon binding to the apo-protein has been extensively studied.

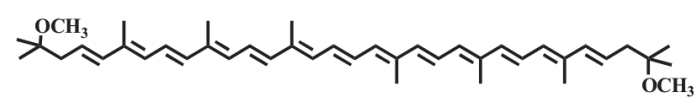

Figure 1. The Chemical structure of all-trans spirilloxanthin.

\section{MATERIALS AND METHODS}

Sample preparation. Cultivation and harvesting of the cells of a purple photosynthetic bacterium, Rsp. rubrum wild-type strain S1, were performed as previously described (Nakagawa et al., 2008b; 2009). All-trans-spirilloxanthin, the LH1 subunit-type complexes (B820 complexes) and the native LH1 complexes were isolated from Rsp. rubrum S1 and purified as reported previously (Nakagawa et al., 2008a, 2009). Reconstitution (re-association) of the LH1 complexes $\left(\mathrm{REC}_{\mathrm{spx}}\right)$ were performed using the LH1 subunit-type complex and the purified all-trans spirilloxanthin as reported previously (Nakagawa et al., 2008a,b).

The LH1 complexes thus obtained were dispersed in $20 \mathrm{mM}$ Tris. $\mathrm{Cl}$ (pH 8.0) buffer solution containing 0.01\% lauryldimethylamine-oxide (LDAO), and then uniformly dispersed into polyvinyl alcohol (PVA) film as reported previously (Nakagawa et al., 2008a; 2008b). Briefly, $50 \mathrm{mg}$ of PVA was added in $1 \mathrm{ml}$ solution of LH1 at the concentration of $\mathrm{OD}_{880 \mathrm{~nm}}$ about 10 , and stirred overnight at $4^{\circ} \mathrm{C}$ to dissolve PVA. The solution was dropped onto the surface of gold electrodes vapor-deposited on the glass surface and dry up under reduced pressure.

Spectroscopic measurements. Absorption spectra were recorded using a commercially available UV-VIS spectrophotometer (V-670, JASCO) as well as the homebuilt set-up for Stark absorption measurements described below.

Figure 2 shows the block diagram of the home-built set-up for Stark absorption measurements. The details of this set-up have already been reported (Yanagi et al., 2004). For the measurements of steady-state absorption spectra using the Stark set-up, a xenon lamp (150W, L2274, Hamamatsu) was replaced by a tungsten-halogen lamp (55W, H3-12V55W, Phoenix) as a light source of white continuum. The light output from the monochromator (SpectraPro 150, Acton Research) passes through

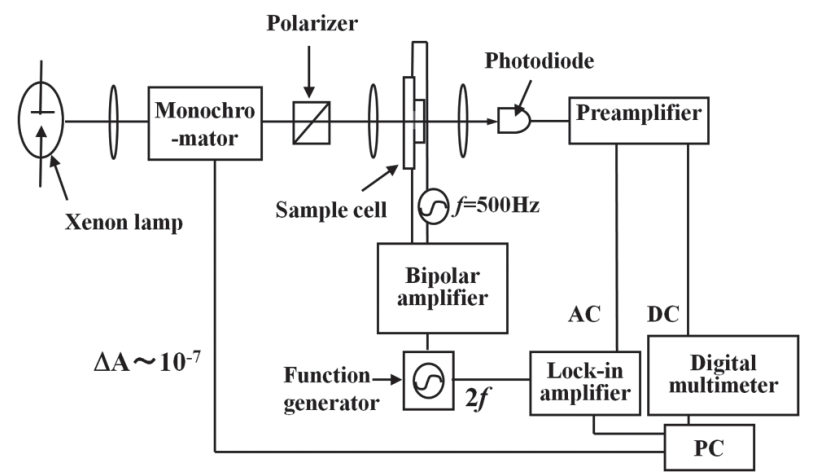

Figure 2. The Block diagram of the set-up for Stark absorption spectroscopy.

a quartz polarizer (Gran-Thomson prism) to make linear polarization. Direction of the polarization was chosen by rotating the polarizer, and hence the polarization angles against the external electric field, $\chi$, was controlled. Stark absorption spectra were recorded and analyzed in the spectral region from $480 \mathrm{~nm}$ to $580 \mathrm{~nm}$ (carotenoid absorption region). All the measurements were performed at room temperature.

Analysis of Stark absorption spectra. The theoretical representation of the Stark absorption spectrum was propounded by Liptay (1974). The Stark absorption spectrum $(\triangle A(v))$ can well be reproduced using the 0 th, 1 st and 2 nd order derivatives of the steady-state absorption spectrum $(A(v))$ as shown in equation (1).

$$
\begin{aligned}
& \Delta \mathrm{A}(v)=\left[\mathrm{A}_{\chi} \cdot \mathrm{A}(v)+\mathrm{B}_{\chi} \cdot \frac{v}{15 h c} \frac{d(\mathrm{~A}(v) / v)}{d v}+\right. \\
& \left.\mathrm{C}_{\chi} \cdot \frac{v}{30 h^{2} c^{2}} \frac{d^{2}(A(v) / v)}{d v^{2}}\right] \cdot\left|f \cdot \mathbf{E}_{\text {ext }}\right|^{2}
\end{aligned}
$$

where $v$ represents the frequency of the incident radiation, $f$ is the correction factor for the local field, $E_{\text {ext }}$ is the externally applied electric field. The constants, $A_{\gamma}$, $\mathrm{B}_{\chi}, \mathrm{C}_{\chi}$, can be determined by spectral fitting. Assuming the contribution of the $A_{c}$ term is negligible and this is mostly hold true for the LH complexes (Yanagi et al., 2004), the coefficients $B_{\chi}$ and $C_{\chi}$ can be written as equations (2) and (3).

$$
\begin{aligned}
& \mathrm{B}_{\chi} \approx \frac{2}{5} \operatorname{Tr}(\Delta \boldsymbol{\alpha})+\left(3 \cos ^{2} \chi-1\right)\left(\frac{3}{2} \mathbf{m} \cdot \boldsymbol{\Delta \alpha} \cdot \mathbf{m}-\operatorname{Tr}(\boldsymbol{\Delta \alpha})\right) \\
& \mathrm{C}_{\chi}=5|\Delta \boldsymbol{\mu}|^{2}+\left(3 \cos ^{2} \chi-1\right)\left(3(\boldsymbol{m} \cdot \Delta \boldsymbol{\mu})^{2}-|\Delta \boldsymbol{\mu}|^{2}\right)
\end{aligned}
$$

Here $m$ shows the unit vector of the transition dipole moment. Nonlinear optical parameters, $m \cdot \Delta \alpha \cdot m, \operatorname{Tr}(\Delta \alpha)$, $m \cdot \Delta \mu$ and $|\Delta \mu|$, can be determined by the fitting of the curves drawn by equations (2) and (3) to the experimentally determined values of $\mathrm{B}_{\chi}$ and $\mathrm{C}_{\chi}$ for $\chi=0^{\circ}, 30^{\circ}$, $54.7^{\circ}, 70^{\circ}$ and $90^{\circ}$. The angles of $\Delta \alpha$ and $\Delta \mu$ with respect to the direction of $\mathrm{m}, \theta_{\Delta \alpha}$ and $\theta_{\Delta \mu}$, respectively, can be determined by the following equations.

$$
\begin{gathered}
\frac{\boldsymbol{m} \cdot \Delta \boldsymbol{\alpha} \cdot \boldsymbol{m}}{\operatorname{Tr}(\Delta \alpha)} \approx \cos \theta_{\Delta \alpha} \\
\frac{\boldsymbol{m} \cdot \Delta \boldsymbol{\mu}}{|\Delta \boldsymbol{\mu}|}=\cos \theta_{\Delta \mu}
\end{gathered}
$$




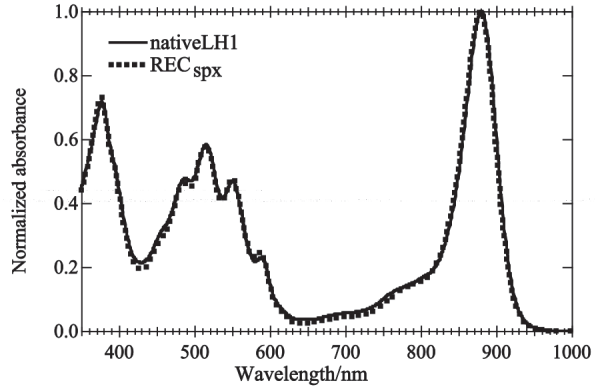

Figure 3. Absorption spectra of the native (solid line) and reconstituted (REC ${ }_{\mathrm{SPX}}$, dotted line) LH1 complexes in PVA films at room temperature.

\section{RESULTS AND DISCUSSION}

\section{Steady-state absorption spectra}

Figure 3 shows the steady-state absorption spectra of the native and reconstituted $\left(\mathrm{REC}_{\mathrm{spx}}\right)$ LH1 complexes in the PVA films. Both spectra show basically the same profiles. Characteristic absorption band from 450 to 470 $\mathrm{nm}$ can be assigned to the $\mathrm{S}_{2} \leftarrow \mathrm{S}_{0}$ transition of spirilloxanthin bound to the LH1 complexes. The bands peaking at 377,580 , and $878 \mathrm{~nm}$ were assigned to the Soret, $Q_{x}$ and $\mathrm{Q}_{\mathrm{y}}$ bands of $\mathrm{BChl} a$, respectively.

\section{Stark absorption spectra}

Figure 4 shows the Stark absorption spectra at $\chi=$ $54.7^{\circ}$ (open circles) and the results of fittings (thin lines) for (a) native and (b) reconstituted LH1 complexes in PVA films. The 0th, 1st and 2nd order derivatives of the steady-state absorption spectra that contribute to the fittings were also shown in the Figure. The Stark absorption spectra were well reproduced by these fittings. The $0^{\text {th }}$ derivatives contribute relatively small and negatively to the Stark absorption spectra. This supports the assumption for equations (2) and (3), where the transition dipole-moment polarizability assumed to be negligible (Bublitz et al. 1997b).

Figure 5 shows the plots of $\mathrm{B}_{\gamma}$ and $\mathrm{C}_{\gamma}$ determined by the fittings using equation (1) against $\chi$ for the native (open triangles) and reconstituted (open circles) LH1 complexes in PVA films. The results of the fittings using equations (2) and (3) were also shown in solid lines. Table 1 summarizes the nonlinear optical parameters determined in this study. Small difference in the values of $\operatorname{Tr}(\Delta \alpha)$, between the native and reconstituted LH1 complexes should be converged after iterate experiments.

The $|\Delta \mu|$ values were determined to be $7.7 \pm 0.5$ and $7.0 \pm 0.6$ for the native and reconstituted LH1 complexes, respectively. These values are in good agreement with the previous report (Nakagawa et al., 2008a). The change of static dipole moment upon photoexcitation $(\Delta \mu)$ is written by the following equation (Bublitz et al., 1997b; Nakagawa et al., 2008a).

$\Delta \mu=\Delta \alpha \cdot E+\Delta \mu_{\mathrm{i}}$

A molecule having strict center of symmetry, like alltrans spirilloxanthin, should not have any values of static dipole moment $\left(\mu_{i}\right)$. Therefore in this case the change in the static dipole moment $(\Delta \mu)$ should solely come from the contribution of the induced dipole moment $(\Delta \alpha \cdot E)$. Carotenoid molecules typically show large $\Delta \mu$ values (Gottfried et al., 1991; Krawczyk et al., 1995). This is thought to be due to the contribution of the induced (a)
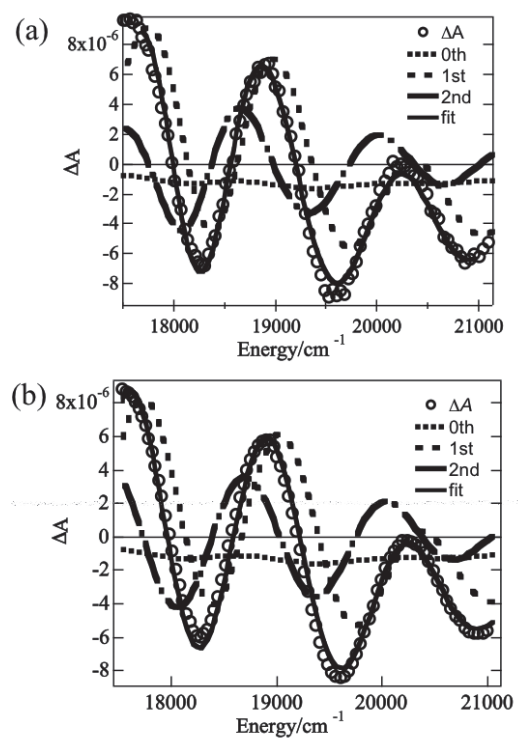

Figure 4. Stark absorption spectra at $X=54.7^{\circ}$ (open circles) (a) native and (b) reconstituted LH1 complexes in PVA films with the results of spectral fitting (thin solid lines).

Spectra responsible for the $\mathrm{S}_{2} \leftarrow \mathrm{S}_{0}$ absorption region of spirilloxanthin are shown. The contributions of the 0th (dotted lines), 1st (broken lines) and 2nd (thick solid lines) order derivatives of the original absorption spectra are also shown for comparison.

dipole moment that is produced by the effect of surrounding matrix molecules. In the previous report $(\mathrm{Na}-$ kagawa et al., 2008a), we have thus assumed that the alltrans spirilloxanthin bound to the LH1 complex shows the large $\Delta \mu$ value, even though it conserves the center of symmetry. If this assumption is correct, the angles of $\theta_{\Delta \alpha}$ and $\theta_{\Delta \mu}$ of all-trans spirilloxanthin bound to the LH1 complex should show exactly the same values since $\mu_{i}$ term (intrinsic static dipole moment) equals to zero in equation (6). Actually this holds true in the cases of $\mathrm{REC}_{\mathrm{SPX}}$ (see Table 1) and all-trans lycopene isotropically fixed in a solvent matrix (Krawczyk et al., 2006).

However, the native LH1 complex shows slight but different values of $\theta_{\Delta \alpha}$ and $\theta_{\Delta \mu}$ even though the experimental errors taken into consideration (see Table 1). This is a marked and new finding that relates to discuss the molecular structure of carotenoid bound to the native LH1 complexes. The cause of the different $\theta_{\Delta \alpha}$ and $\theta_{\Delta \mu}$ values is thought to be due to the contribution of the $\mu_{i}$ term in equation (6). The $\mu_{i}$ value can be generated when the molecule loses its center of symmetry. Therefore, the present finding suggests that the molecular structure of all-trans spirilloxanthin is somehow deformed so as to lose its center of symmetry according to pigment-protein interaction when bound to the native LH1 complexes.

Table 1. Nonlinear optical parameters determined for the native and reconstituted LH1 complexes in PVA.

The parameters were determined from the fittings of Stark absorption spectra observed in different $X$ values.

\begin{tabular}{lcc}
\hline Carotenoid & Native & RECspx \\
\hline $\operatorname{Tr}(\Delta a)\left[\AA^{3} / \mathrm{f}^{3}\right]$ & $1700 \pm 200$ & $1480 \pm 200$ \\
$|\Delta \mu|[\mathrm{D} / \mathrm{f}]$ & $7.7 \pm 0.5$ & $7.0 \pm 0.6$ \\
$\theta_{\Delta \alpha}\left[^{\circ}\right]$ & $28 \pm 2$ & $30 \pm 1$ \\
$\theta_{\Delta \mu}\left[^{\circ}\right]$ & $36 \pm 2$ & $30 \pm 1$ \\
\hline
\end{tabular}




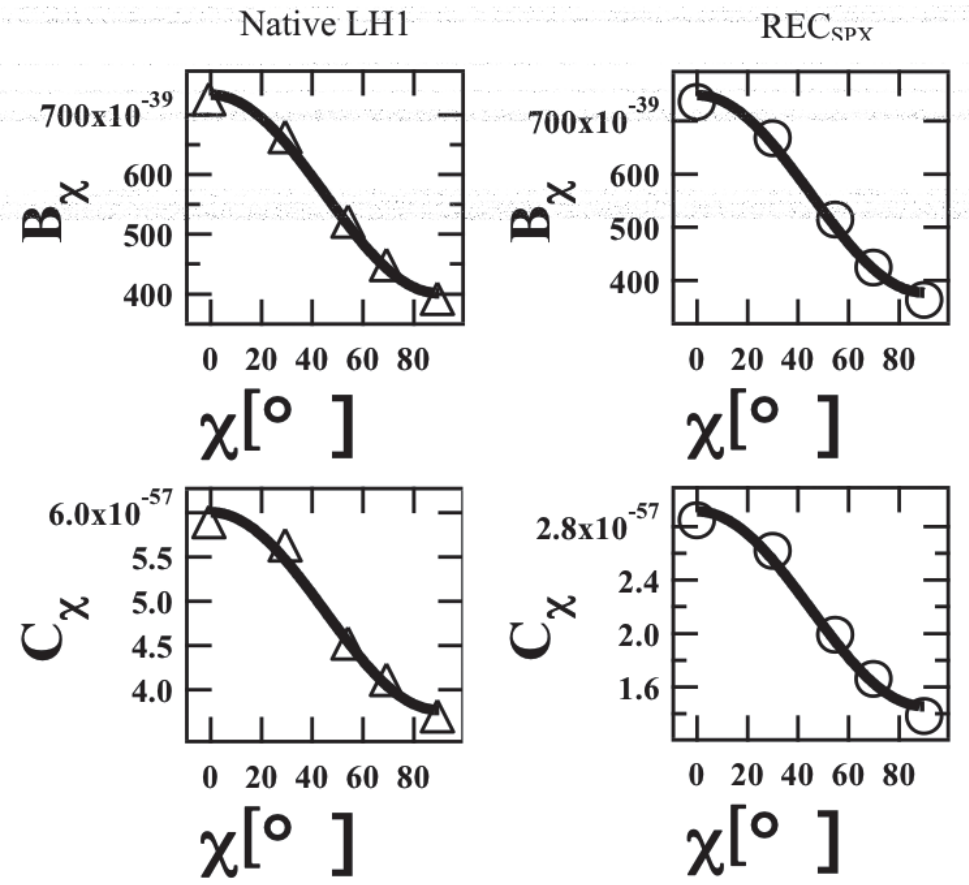

Figure 5. Plots of the values of $B_{x}$ (upper panel) and $C_{x}$ (lower panel) for different angles of $X(x=$ $0,30,54.7,70$ and $90^{\circ}$ ) for native (circles) and reconstituted ( $\mathrm{REC}_{\mathrm{SPX}}$, triangles) LH1 complexes. Results of the theoretical fittings are also shown in solid lines.

\section{CONCLUSION}

The polarization angle dependence of the Stark absorption spectra of all-trans spirilloxanthin bound to the native and reconstituted LH1 complexes have been investigated. The large $\Delta \mu$ value of spirilloxanthin bound to the reconstituted LH1 complex can be explained solely due to the effect of induced dipole moment. This supports the validity of the assumption of the symmetric molecular structure of spirilloxanthin bound to the reconstituted LH1 complex. However, the native LH1 complex shows slightly different values of $\theta_{\Delta \alpha}$ and $\theta_{\Delta \mu}$ and hence suggests the presence of asymmetrical deformation of all-trans spirilloxanthin when bound to the native LH1 complex. This present study shows the usefulness of Stark absorption spectroscopy to discuss the molecular structure of carotenoids bound to the pigmentprotein complexes.

\section{Acknowledgement}

$\mathrm{HH}$ thanks Nissan Science Foundation and HFSP for financial support. RJC thanks BBSRC and HFSP for financial support.

\section{REFERENCES}

Akahane J, Rondonuwu FS, Fiedor L, Watanabe Y, Koyama Y (2004) Dependence of singlet-energy transfer on the conjugation length of carotenoids reconstituted into the LH1 complex from Rhodospirillum rubrum G9. Chem Phys Lett 393: 184-191.

Bublitz GU, Boxer SG (1997a) Stark spectroscopy: Applications in chemistry, biology, and materials science. Ann Rev Phys Chem 48: 213-242.

Bublitz GU, Ortiz R, Marder SR, Boxer SG (1997b) Stark spectroscopy of donor/acceptor substituted polyenes. J Am Chem Soc 119: 3365-3376.

Cogdell RJ, Gall A, Köhler J (2006) The architecture and function of the light-harvesting apparatus of purple bacteria: from single molecules to in vivo membranes. Q Rev Biophys 39: 227-324.

Cogdell RJ, Gardiner AT, Gabrielsen M, Southall J, Roszak AW, Isaacs NW, Fujii R, Hashimoto H (2008) The structure of purple bacterial antenna complexes. In Photosynthetic Protein Complexes: A Structural Approach, Fromme P ed, pp 325-340. Wiley-Blackwell, Weinheim, Germany.
Gottfried DS, Steffen MA, Boxer SG (1991) Large protein-induced dipoles for a symmetrical carotenoid in a photosynthetic antenna complex. Science 251: 662-665.

Iida K, Inagaki JI, Shinohara M, Suemori Y, Ogawa M, Dewa T, Nango M (2005) Near-IR absorption and fluorescence spectra and AFM observation of the light-harvesting 1 complex on a mica substrate refolded from the subunit light-harvesting 1 complexes of photosynthetic bacteria Rhodospirillum rubrum. Langmuir 21: 3069-3075.

Ke B (2001) Photosynthesis: Photobiochemistry and Photobiophysics, pp 1-46. Kluwer Academic Publishers, Dordrecht, The Netherlands.

Krawczyk BJ, Luchowski R, Wiącek D (2006) Electroabsorption spectra of carotenoid isomers: Conformational modulation of polarizability vs. induced dipole moments. Chem Phys 326: 465-470.

Krawczyk S, Daniluk A (1995) Solvent effects and vibrational dependence in electrochromic spectra of carotenoids. Chem Phys Lett 236: 431-437.

Liptay W (1974), Dipole moments and polarizabilities of molecules in excited electronic states. In Excited States. Lim EC ed, vol. 1, pp 129-229. Academic Press, New York, USA

Middendorf TR, Mazzola LT, Lao KQ, Steffen MA, Boxer SG (1993) Stark-effect (electroabsorption) spectroscopy of photosynthetic reaction centers at $1.5 \mathrm{~K}$ - evidence that the special pair has a large excited-state polarizability. Biochim Biophys Acta 1143: 223-234.

Nakagawa K, Nakano T, Fukui N, Nakashima A, Sakai A, Mizuno T, Dewa T, Iida K, Gardiner AT, Cogdell RJ, Fujii R, Hashimoto H, Nango M (2009) Reconstitution of the light-harvesting 1 (LH1) Complex Using LH1-a and LH1-b polypeptides, separately isolated from the purple photosynthetic bacterium Rhodospirillum rubrum, together with bacteriochlorophyll $a$ and all-trans carotenoids. Carotenoid Science 14: 54-57.

Nakagawa K, Suzuki S, Fujii R, Gardiner AT, Cogdell RJ, Nango M, Hashimoto $H$ (2008a) Probing the effect of the binding site on the electrostatic behavior of a series of carotenoids reconstituted into the light-harvesting 1 complex from purple photosynthetic bacterium Rhodospirillum rubrum detected by stark spectroscopy. J Phys Chem B 112: 3467-9475.

Nakagawa K, Suzuki S, Fujii R, Gardiner AT, Cogdell RJ, Nango M, Hashimoto H (2008b) Probing binding site of bacteriochlorophyll a and carotenoid in the reconstituted LH1 complex from Rhodospirillum rubrum S1 by Stark spectroscopy. Photosynth Res 95: 339-344.

Roszak AW, Howard TD, Southall J, Gardiner AT, Law CJ, Isaacs NW, Cogdell RJ (2003) Crystal structure of the RC-LH1 core complex from Rhodopseudomonas palustris. Science 302: 1969-1972.

Yanagi K, Hashimoto H, Gardiner AT, Cogdell RJ (2004) Stark spectroscopy on the LH2 complex from Rhodobacter sphaeroides strain G1C; frequency and temperature dependence. J Phys Chem B 108: 10334-10339. 